\title{
Design of LIDAR wide field of view receiver based on MEMS micro- mirror and high sensitivity APD (Erratum)
}

Xichen Wang, Pallab Choudhury, Hengheng Liang, Yuhang Wang, Ligong Chen

Xichen Wang, Pallab K. Choudhury, Hengheng Liang, Yuhang Wang, Ligong Chen, "Design of LIDAR wide field of view receiver based on MEMS micromirror and high sensitivity APD (Erratum)," Proc. SPIE 11781, 4th Optics Young Scientist Summit (OYSS 2020), 1178127 (11 May 2021); doi: $10.1117 / 12.2600830$

SPIE. Event: Optics Frontier: Optics Young Scientist Summit, 2020, Ningbo, China 


\title{
Design of UDAR wide field of view receiver based on MEMS mic ro-minor and high sensitivity APD (Enratum)
}

\author{
Xichen Wang, Hengheng Liang, and Ligong Chen \\ Chongqing Univ. of Technology (China) \\ Pallab K. Choudhury \\ Khulna Univ. of Engineering \& Technology (Bangladesh) \\ Yuhang Wang \\ J iangsu Univ. of Science and Technology (China) \\ Proceedings Volume 11781, 4th Optics Young Sc ientist Summit (OYSS 2020); 117811U (2021) \\ https://doi.org/10.1117/12.2600830
}

Event 4th Optics Young Sc ientist Summit (OYSS 2020), 2021, Ning bo, China

Online Publication Date: 28 February 2021

Enatum Published: 10 May 2021

A revised version of this manuscript was published on 10 May 2021. Deta ils of the revision a re provided in the text that a ccompa nies this Erratum. The original paper has been updated. 